\title{
Nanophotonics: Fabrications and Application of Nanoscale Optics to Novel Photonic Devices
}

\author{
Takashi Yatsui, ${ }^{1}$ Chennupati Jagadish, ${ }^{2}$ and Gilles Lerondel ${ }^{3}$ \\ ${ }^{1}$ School of Engineering, University of Tokyo, Bunkyo-ku, Tokyo 113-8656, Japan \\ ${ }^{2}$ Department of Electronic Materials Engineering, Research School of Physics and Engineering, \\ Australian National University, Canberra, ACT 0200, Australia \\ ${ }^{3}$ Laboratoire de Nanotechnologie et d'Instrumentation Optique, Institut Charles Delaunay, CNRS UMR 6281, \\ Université de Technologie de Troyes, CS 42060, 10004 Troyes Cedex, France \\ Correspondence should be addressed to Takashi Yatsui; yatsui@ee.t.u-tokyo.ac.jp
}

Received 27 November 2014; Accepted 27 November 2014

Copyright (C) 2015 Takashi Yatsui et al. This is an open access article distributed under the Creative Commons Attribution License, which permits unrestricted use, distribution, and reproduction in any medium, provided the original work is properly cited.

Nanophotonics is a generic technology that can overcome the deadlock of conventional photonics, in which the size is limited by diffraction limits of light. Based on interdisciplinary studies including condensed-matter physics, optical science, and quantum field theory, nanoscale materials and optical energy transfer at nanoscale have been extensively studied. The recent development on nanoscale optics opens up new concept of nanoscale optical theory, as an example dressed photon theory, and a variety of new phenomena have been investigated. The results of the basic research have been applied to numerous applications, including nanometersized photonic devices, nanoscale fabrication techniques, and highly efficient energy conversion. Here, we have invited a few papers that address the above topics.

One paper of this special issue addresses solution searching devices based on the optical near-field energy transfer between quantum dots (QDs). The results shown here indicated the possibility of controlling the spatiotemporal dynamics between multiple QDs, resulting in a higher performance of solution searching devices. Another paper reported the new type of light emitting device (LED) of silicon $(\mathrm{Si})$. Even though $\mathrm{Si}$ is an indirect band gap material, they realized Si based LED by introducing dressed photon and phonon (DPP) assisted annealing process. Moreover, the emitting spectrum was controlled by DPP assisted annealing using a short pulse pair. One of the papers also addressed LED but based on GaP, which is also an indirect band gap semiconductor. In this paper, GaP based LED fabricated by DPP assisted annealing process is reported. Such device has unique emitting properties depending on the annealing process. Another paper demonstrated the possibility of high-contrast switching of local surface plasmon using hybrid structure made of metallic nanorods and a phase change material. Additionally, the authors suggested the one-dimensional cellular automata algorithm using metallic nanorod and phase change material. Another paper reported the unique flattening technique made possible thanks to DPP. By using DPP etching, selective flattening of the smallest surface features is made possible, resulting in an angstrom scale ultraflat plastic substrate.

Takashi Yatsui Chennupati Jagadish Gilles Lerondel 

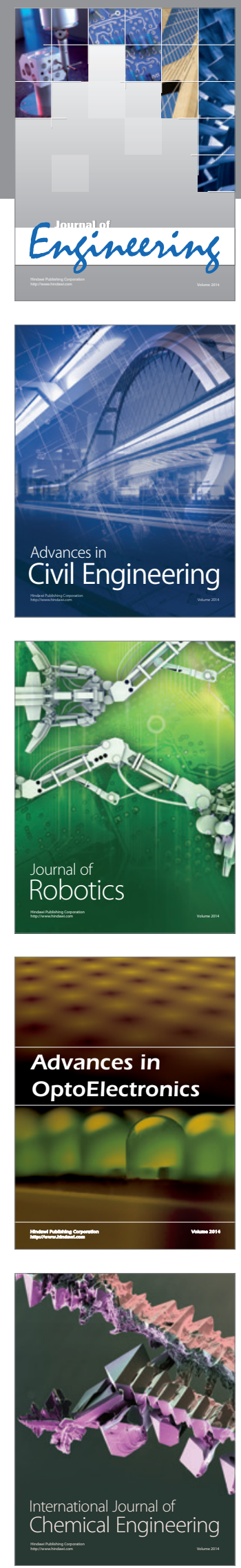

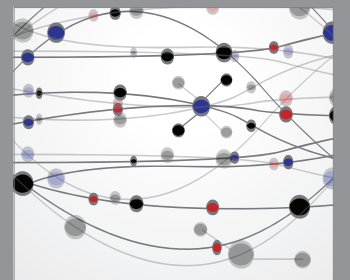

The Scientific World Journal
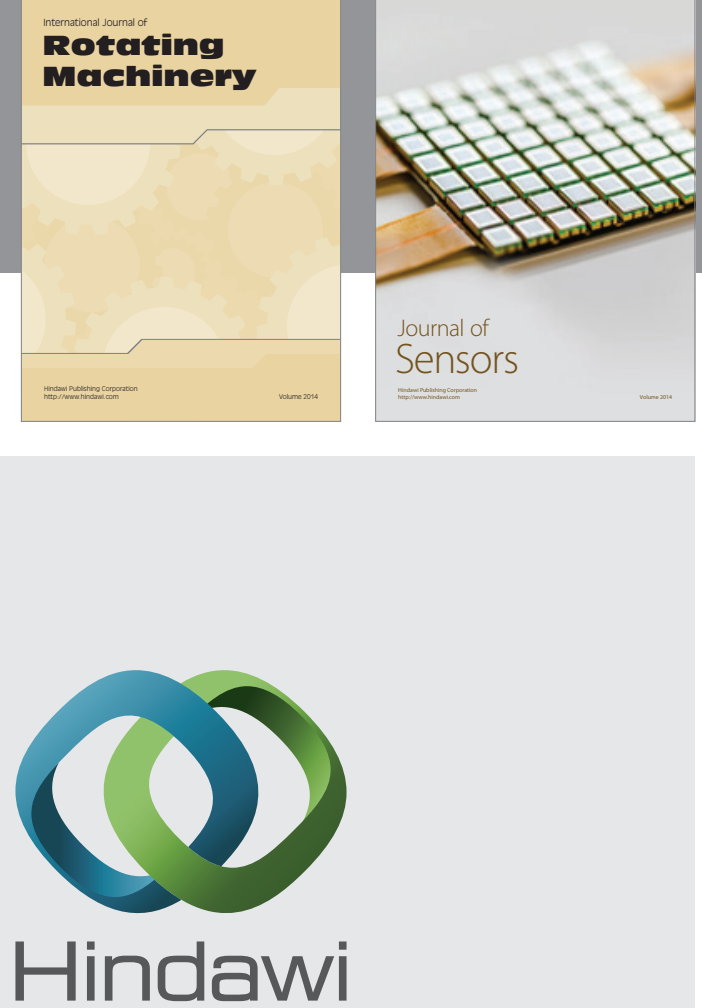

Submit your manuscripts at http://www.hindawi.com
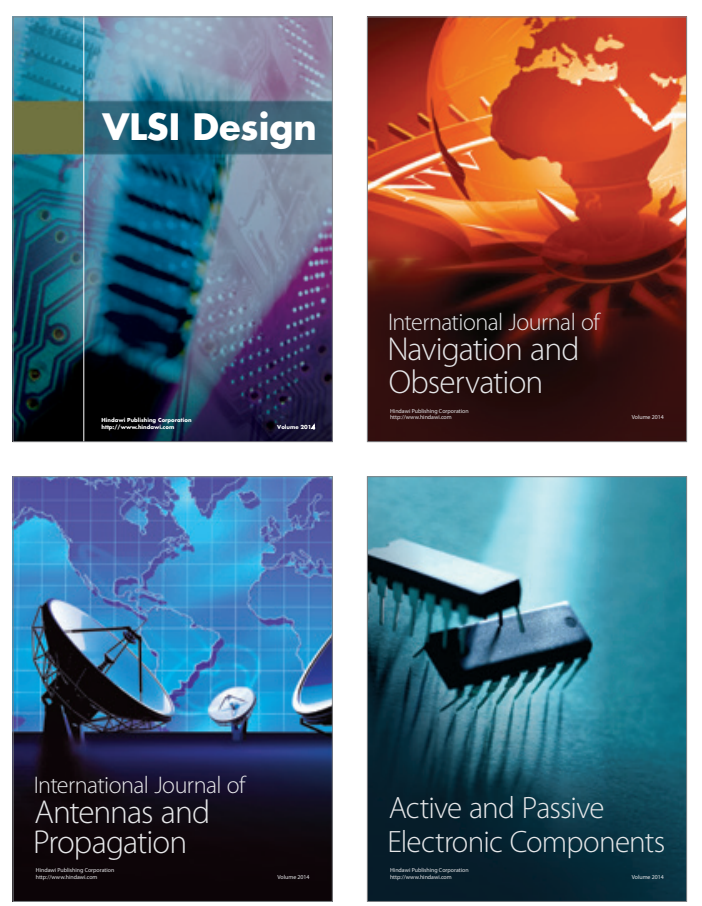
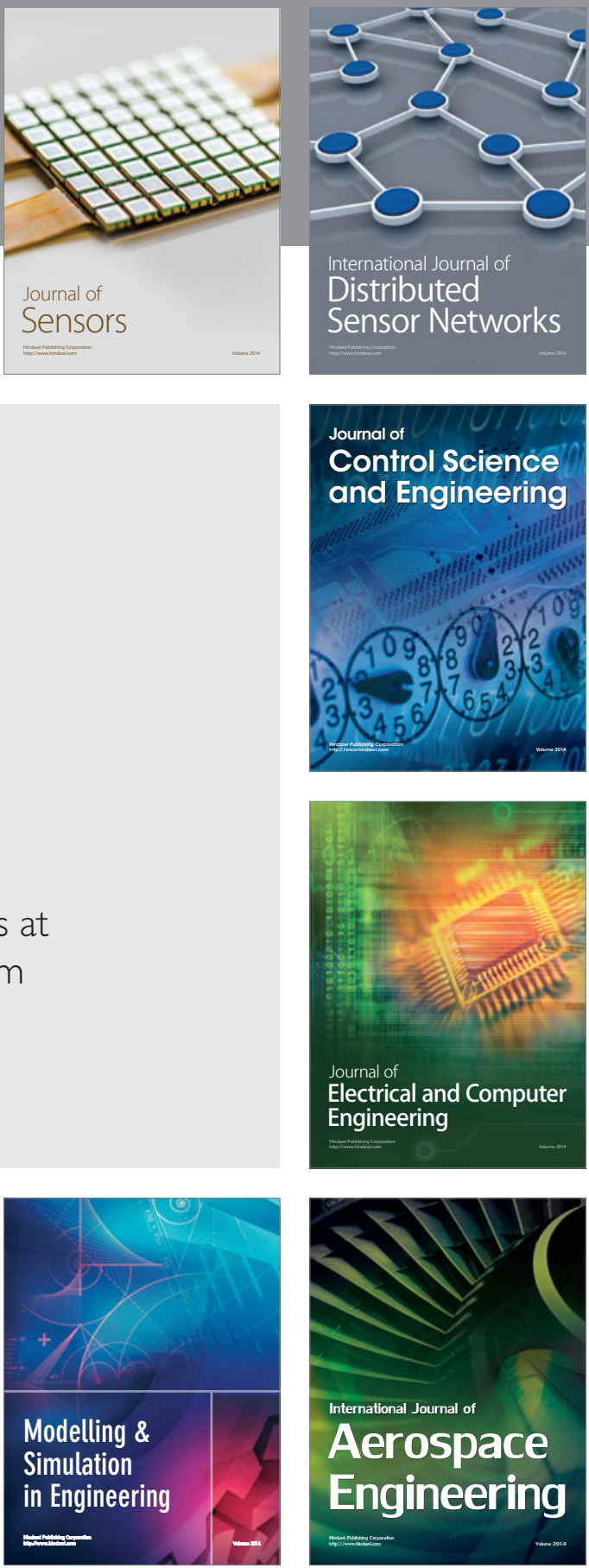

Journal of

Control Science

and Engineering
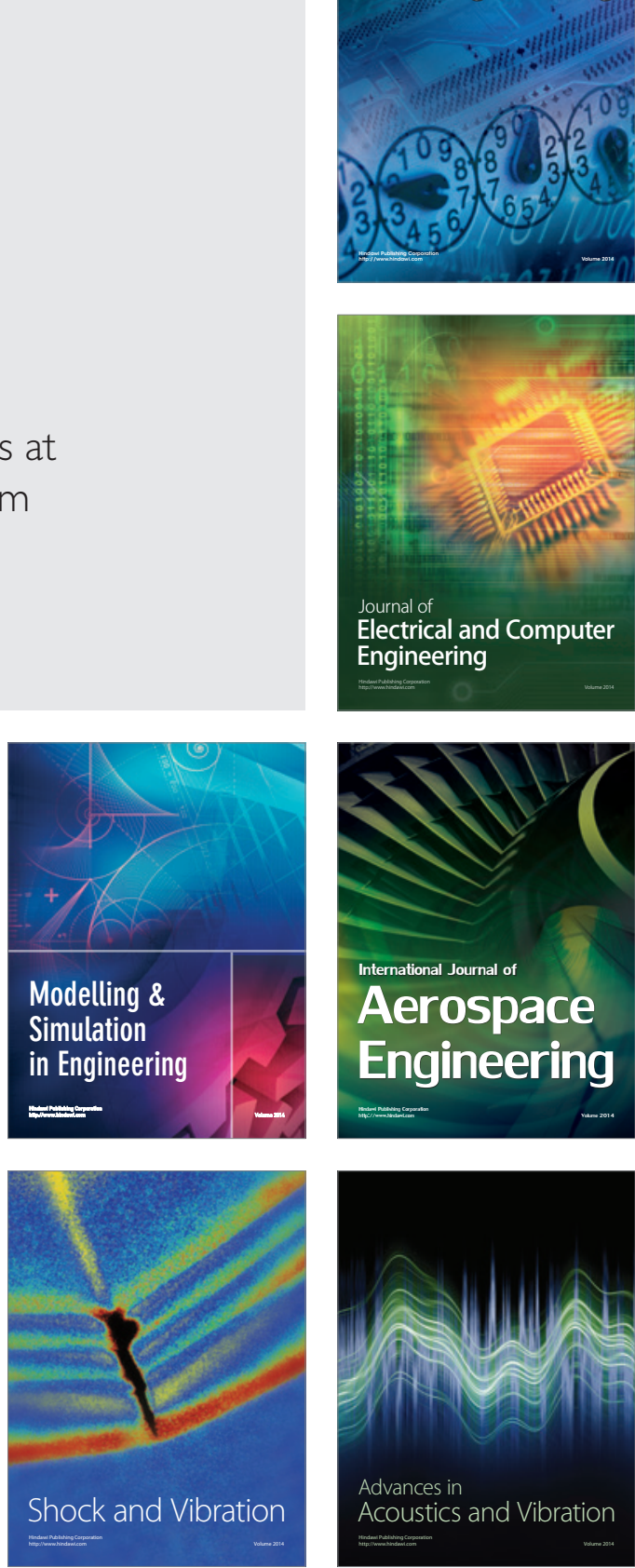